\title{
Prediction of impaired metabolic adaptation by antenatal Doppler studies in small for gestational age fetuses
}

\author{
J M Hawdon, M P Ward Platt, S McPhail, H Cameron, S A Walkinshaw
}

\begin{abstract}
The increased risk of hypoglycaemia for infants who are small for gestational age (SGA) is well known, and further information regarding metabolic adaptation can be gained by examining the profiles of glucose and other metabolic fuels in such infants. Not all SGA infants experience hypoglycaemia, however, and the ability to identify, antenatally, those at most risk would be helpful for obstetric and neonatal management. This study investigated metabolic differences between two groups of SGA infants who had antenatal umbilical artery Doppler studies. Fourteen had end diastolic velocities (EDV) present and 11 had absent EDV.
\end{abstract}

The absent EDV group had significantly lower non-esterified fatty acid (NEFA) concentrations at birth, and lower blood glucose and NEFA concentrations in the first six hours after birth. After the first day, few metabolic differences were found between the two groups.

Antenatal Doppler studies may identify those SGA infants who fail to achieve neonatal metabolic adaptation and are most at risk of early neonatal hypoglycaemia.

The healthy fetus receives a constant supply of nutrients from its mother via the placental circulation. Even at times of maternal fasting, fetal blood glucose concentrations are usually maintained as a result of metabolic changes in the fetus itself. After birth, the healthy neonate responds to the cessation of intrauterine nutrition by mobilising glycogen stores to produce glucose, and by initiating gluconeogenesis from amino acids and other substrates. Lipolysis and $\beta$-oxidation of fatty acids provide alternative fuels to glucose and the energy necessary for gluconeogenesis.

It is well known that infants who are small for gestational age (SGA) are less able to achieve the metabolic adaptation required of them at birth and are at risk of hypoglycaemia $^{1} 2$ and, in some cases, hyperglycaemia. ${ }^{3}$ We also have found differences between SGA and normal infants in terms of ketone body production and the ability to utilise gluconeogenic precursors in the early neonatal period. ${ }^{4}$ The clinical implications of such reduced availability of metabolic fuels are not yet clear, but recent studies have suggested that neonatal blood glucose concentra- tions below $2.6 \mathrm{mmol} / \mathrm{l}$ are associated with adverse neurological effects, especially for preterm infants. ${ }^{56}$

Some metabolic effects of being SGA may be evident antenatally, such as hypoxia, acidosis, hyperlactataemia, and hypoglycaemia. $^{7} 8$

However, not all SGA infants experience failure of metabolic adaptation and it would be of obstetric, paediatric, and maternal benefit to identify those fetuses most at risk. Male SGA infants in particular are thought to be most at risk of neonatal hypoglycaemia, ${ }^{9}$ and it appears that the size and relative body proportions of the infant affect vulnerability. 910 Doppler ultrasound studies of the umbilical vessels have suggested that this antenatal test can identify SGA fetuses at risk of significant morbidity and mortality. ${ }^{11}$

The purpose of the present study was to compare the metabolic changes seen after birth in a group of SGA infants for whom end diastolic velocities were present on antenatal Doppler study, and a group of equally SGA infants for whom end diastolic velocities (EDV) were absent, to investigate whether abnormal Doppler studies predicted failure of metabolic adaptation. If this hypothesis were true, Doppler studies would then add information to that available from fetal measurements, and might influence clinical management of such small fetuses.

\section{Subjects and methods}

Twenty five normally formed singleton infants of mixed gestational age, identified by antenatal ultrasound assessment of fetal weight as being SGA, and who subsequently had birth weights that were more than two standard deviations below the mean for gestational age, were studied. Fetal umbilical Doppler ultrasound studies were carried out using continuous wave Doppler ultrasound (Doptek) with a $100 \mathrm{~Hz}$ filter. Waveforms typical of the fetal umbilical artery were identified and included continuous flow consistent with umbilical venous flow in the opposing channel. The umbilical artery was examined at three different anatomical areas. In all cases, the pulsatility index ( $A-B /$ mean) was calculated for at least five cycles and the results from the three areas averaged. The last examination carried out within five days of delivery was used to classify the fetus. The result was considered abnormal when EDV were absent (11 fetuses), and normal when EDV were present (14 fetuses). 
Umbilical venous blood was taken at delivery and infants were sampled before their first feed or institution of intravenous glucose infusion. Intravenous and enteral fluids were given according to nursery protocols; in order to standardise conditions during sampling, each infant received no enteral feed and no more than $5 \mathrm{mg} / \mathrm{kg} /$ minute glucose intravenously for at least one hour before sampling. Samples were taken, once a day for the first postnatal week, by heelprick, the foot being sufficiently warm to allow free blood flow: $40 \mu \mathrm{l}$ blood were added to $3 \%$ perchloric acid to denature enzyme activity; $50 \mu \mathrm{l}$ plasma were also collected. Samples were analysed, using a Cobas fast centrifugal autoanalyser for glucose, gluconeoganic precursors (pyruvate, alanine, lactate, glycerol), ketone bodies ( $\beta$ hydroxybutyrate and acetoacetate) and nonesterified fatty acids (NEFA).

Each infant's birth weight, head circumference (HC) and mid-arm circumference (MAC) was recorded. A birthweight SD score was calculated, which represented the number of SDs by which the infant's birth weight differed from the mean for gestational age. Birthweight centile charts used were those previously derived from Newcastle births. ${ }^{12}$

Data were analysed using the statistical package for the social sciences (SPSS X). $\chi^{2}$ Tests were used to compare background data for groups of infants. As the blood metabolite concentrations did not have a normal distribution, Mann-Whitney U tests were used to compare metabolite concentrations between groups.

Ethical approval for the study was granted by the ethics committee of Newcastle Health Authority. Informed consent was obtained from parents for the inclusion of each baby in the study, and, as far as possible, sampling was coordinated with clinically necessary tests.

\section{Results}

Details of the groups of infants are given in table 1 . There were no significant differences

Table 1 Details of infants studied

\begin{tabular}{|c|c|c|c|}
\hline & $\begin{array}{l}\text { Absent EDV } \\
(n=11)\end{array}$ & $\underset{(n=14)}{\text { Present }} E D V$ & p Value \\
\hline $\begin{array}{l}\text { Median gestation (weeks) } \\
\text { Range } \\
\text { Male:female ratio } \\
\text { Median birth weight (g) } \\
\text { Range } \\
\text { Mean birth weight SD score (SD) } \\
\text { Mean MAC:HC (SD) }\end{array}$ & $\begin{array}{l}35 \\
32-40 \\
8: 3 \\
1525 \\
668-2020 \\
-3 \cdot 22(0 \cdot 90) \\
0 \cdot 24(0 \cdot 02)\end{array}$ & $\begin{array}{l}37 \\
32-40 \\
5: 9 \\
1903 \\
859-2296 \\
-2 \cdot 95(0.54) \\
0 \cdot 26(0.03)\end{array}$ & $\begin{array}{l}0.076 \\
0.151 \\
0.065 \\
0.389 \\
0.551\end{array}$ \\
\hline & $\begin{array}{l}\text { Absent } E D V \\
(n=11)\end{array}$ & & $\begin{array}{l}\text { Present } E D V \\
(n=14)\end{array}$ \\
\hline $\begin{array}{l}\text { Cord blood at delivery: } \\
\text { Mean pH (SD) } \\
\text { Median NEFA (range) } \\
\text { Median ketone bodies (range) } \\
\text { Median TGS (range) } \\
\text { Median lactate (range) } \\
\text { Median glucose (range) }\end{array}$ & $\begin{array}{l}7 \cdot 31(0.07) \\
0.08(0.02-0.15) \\
0.05(0.03-0.16) \\
3.34(1.33-5 \cdot 57) \\
2 \cdot 70(0.98-4 \cdot 89) \\
3.0(2 \cdot 5-4 \cdot 2)\end{array}$ & & $\begin{array}{l}7.31(0.10) \\
0.31(0.04-0.65)^{* *} \\
0.17(0.06-0.36)^{*} \\
4.06(1.50-6 \cdot 70) \\
3.58(1.13-5 \cdot 85) \\
3.9(2 \cdot 5-5 \cdot 8)\end{array}$ \\
\hline $\begin{array}{l}\text { Heelprick blood at <6 hours: } \\
\text { Median NEFA (range) } \\
\text { Median ketone bodies (range) } \\
\text { Median TGS (range) } \\
\text { Median lactate (range) } \\
\text { Median glucose (range) }\end{array}$ & $\begin{array}{l}0.18(0.01-0.32) \\
0.04(0.01-0.08) \\
3.57(1.33-9.91) \\
2.89(0.98-8.40) \\
2.0(1.5-2 \cdot 3)\end{array}$ & & $\begin{array}{l}0.85(0.02-1 \cdot 20)^{*} \\
0.05(0.01-0.11) \\
3.95(1.50-8.55) \\
3.28(1 \cdot 13-7.40) \\
3.0(2 \cdot 0-5 \cdot 2)^{* * *}\end{array}$ \\
\hline
\end{tabular}



Figure 1 Blood glucose concentrations in infants with present or absent EDV.

between the groups in terms of birth weight, gestation, or incidence of obstetric or perinatal problems (such as maternal smoking, instrumental delivery, cardiotocographic evidence of fetal distress, or infant's requirement for active resuscitation after birth). In particular, the severity of growth retardation, as measured by mean birthweight SD score and mean MAC:HC ratio, was similar for both groups. Once enteral or intravenous feeding was established, both groups received intravenous glucose and enteral feeds at similar rates on each postnatal day.

Results of metabolic investigations are shown in table 2. Infants of the absent EDV group had lower blood glucose concentrations at birth and in the first six hours after birth than those of the present EDV group (fig 1, some points are superimposed). Although this difference did not reach statistical significance for cord blood $(p=0.08)$, the difference was highly significant subsequently $(p<0.01)$. Concentrations of ketone bodies and non-esterified fatty acid were significantly lower for the absent EDV group at birth compared with the present EDV group, and NEFA concentrations were lower in the first six hours after birth (fig 2, some points are superimposed). For all infants,



Figure 2 NEFA concentrations in infants with present or absent $E D V$. 
concentrations of lactate and gluconeogenic substrates were high, but there were no differences between the present and absent EDV groups. After the first day there were no metabolic differences between the groups nor in the relationships between metabolic concentrations (ketone bodies/glucose; ketone bodies/NEFA).

Blood glucose concentration at birth and in the first hours of life was not related to gestational age in this study of 25 infants, considered as a whole, or when the groups were considered separately.

None of the infants, in either group, had symptoms of hypoglycaemia, namely convulsions or coma, and the two groups were indistinguishable in terms of clinical characteristics and postnatal complications.

\section{Discussion}

SGA infants with abnormal antenatal Doppler studies may be expected to be those at highest risk of metabolic derangement. Studies correlating antenatal fetal umbilical blood sampling and Doppler indices have suggested that the fetus with abnormal indices is more likely to be hypoglycaemic, hypoinsulinaemic, hypoxaemic, hyperlacticaemic and acidotic. ${ }^{13} 14$ The degree of in utero asphyxia correlates with other metabolic derangements such as hypertriglyceridaemia ${ }^{15}$ and alteration in amino acid metabolism. ${ }^{1617}$ The asphyxiated SGA fetus in utero does not mount a NEFA/ketone body response in the face of hypoglycaemia ${ }^{15}$; this abnormality thus predates the failure of such responses postnatally. However in these studies, the fetuses with abnormal Doppler were significantly smaller than those with normal Doppler, which casts doubt over the added predictive value of Doppler ultrasound over conventional assessment of fetal measurement.

The present study demonstrates that even in babies with similar degrees of moderate/ severe growth retardation, umbilical arterial Doppler can distinguish a group of babies with metabolic maladaptation, and this is consistent with recent data on lactate concentrations in utero. ${ }^{13}$ Such babies had significantly lower blood glucose concentrations in the first few hours after birth. However, as fetuses and neonates, these subjects failed to demonstrate a lipolytic response to low blood glucose concentrations. This correlates well with data obtained from antenatal fetal blood sampling, ${ }^{15}$ and demonstrates that maladaptation extends into postnatal life. The data suggest that SGA infants with absent EDV were subject to reduced availability of the metabolic fuels which are important in the neonatal period. This may have had adverse neurological sequelae, even in the absence of major symptoms, but this study did not aim to investigate the clinical associations of such metabolic abnormalities.

On the other hand, it appears from the present study that SGA babies with EDV present on umbilical Doppler studies are likely to have normal metabolic profiles in the early neonatal period.
This study demonstrates the difficulties in identifying a well matched control group with normal Doppler studies, in that SGA fetuses with abnormal umbilical Doppler are likely to undergo elective early delivery. However, in the present study, the differences in gestational age between the groups were small and statistically insignificant. Moreover, the present study and a recent study ${ }^{4}$ failed to find any influence of gestational age on metabolic adaptation in SGA babies. Therefore we feel that the small differences in gestational age were clinically insignificant.

There is evidence, in a recent study of a similar group of SGA infants, that the feeding regimens used were effective in maintaining adequate glucose concentrations. ${ }^{4}$ In the present study, enteral and intravenous intakes were similar between the groups, and, once feeding was started, there were no differences between the groups in blood glucose concentrations or the number of occasions on which low blood glucose concentrations $(\leq 2.6 \mathrm{mmol} / \mathrm{l})$ were found.

A larger study is recommended to determine the sensitivity and specificity of abnormal Doppler studies for evidence of intrauterine hypoglycaemia and postnatal metabolic derangement. For example, study groups of at least 26 each would be required to show a significant difference (with $95 \%$ confidence, SD for blood glucose concentration $0.9 \mathrm{mmol} / \mathrm{l}$ ) of $0.5 \mathrm{mmol} / \mathrm{h}$ in umbilical venous blood glucose concentration between groups of infants with absent and present EDV. In addition, a follow up study of these babies may demonstrate whether long term neurological damage has been sustained.

We conclude that abnormal antenatal Doppler studies (namely absent end diastolic flow) predicted those SGA fetuses who were at increased risk of early postnatal hypoglycaemia, and it is possible that these fetuses had lower glucose concentrations antenatally, with failure to mount an appropriate lipolytic and ketogenic response. Equally important is the observation that the very small fetus (birth weight $>2$ SD below the mean) with normal Doppler studies was more likely to mount the appropriate metabolic adaptive responses. The damaging effects of neuroglycopenia in the absence of alternative fuels (because of poor counter-regulatory ability in these fetuses and neonates) need to be minimised in cases of severe growth impairment, as in this study. Delivery may be appropriate when antenatal Doppler studies are abnormal, provided the risks of preterm delivery do not outweigh the risks of intrauterine metabolic maladaptation. The monitoring of fetal glucose concentrations during delivery may help determine management.

In the first few hours after birth there was continued risk of severe hypoglycaemia and early adequate energy intake had to be ensured. However, after this age, infants with abnormal Doppler studies were at no greater risk of metabolic problems. It may be that provision of adequate parenteral and enteral energy outweighed the adverse effects of such antenatal risk factors. 
Doppler studies predict metabolic maladaptation, and add to the information already available antenatally regarding fetal size. Therefore abnormal antenatal Doppler studies should alert the obstetrician to the fetus who is at risk in utero, and the paediatrician to the infant who will require close monitoring and the prevention of further metabolic stress.

Dr Hawdon was supported by the Scientific and Research Committee of Newcastle Health Authority and by a Foundation for the Study of Infant Deaths Training FellowFoundation for the Study of Infant Deaths Training Fellow-
ship. Mr Walkinshaw was in receipt of a Subspeciality ship. Mr Walkinshaw was in receipt of a Subspeciality
Training Fellowship from the Royal College of Obstetricians Training Fellowship

and Gynaecologists. We thank the parents who gave consent for the inclusion of
their babies in the study, and hospital staff for their support and cooperation.

1 Cornblath M, Odell GB, Levin EY. Symptomatic neonatal hypoglycemia with toxemia of pregnancy. 7 Pediar 1959;55:545-62.

2 Neligan GA, Robson E, Watson J. Hypoglycaemia in the newborn: a sequel of intrauterine malnutrition. Lancet 1963;i: 1282.

3 Lubchenco LO, Bard $\mathrm{H}$. Incidence of hypoglycemia in newborn infants classified by birth weight and gestational newborn infants classified by birt
age. Pediatrics 1971;47:831-8.

age. Pediatrics $1971 ; 47: 831-8$.
4 Hawdon JM, Ward Platt MP, Aynsley-Green A. Metabolic adaptation in small for gestational age neonates. Arch Dis adaptation in small for

5 Lucas A, Morley R, Cole T. Adverse neurodevelopmental outcome of moderate neonatal hypoglycaemia. $B M \mathscr{f}$ 1988;297:1304-8.
6 Koh THHG, Aynsley-Green A, Tarbit M, Eyre JA. Neuronal dysfunction during hypoglycaemia. Arch Dis Child 1988;63:1353-8

7 Soothill PW, Nicolaides KH, Campbell S. Prenatal asphyxia, hyperlacticaemia, hypoglycaemia, and erythroblastosis in growth retarded fetuses. BMF 1987;294:1051-3.

8 Pardi G, Buscaglia M, Ferrazzi E, et al. Cord sampling for the evaluation of oxygenation and acid-base balance in growth retarded human fetuses. Am $₹$ Obstet Gynecol growth retarded

9 Jarai I, Mestyan J, Schultz K, Lazar A, Halasz M, Krassy I. Body size and neonatal hypoglycaemia in intrauterine growth retardation. Early Hum Dev 1977;1:25-38.

10 Haworth JC, Dilling L, Younoszai MK. Relation of blood glucose to haematocrit, birthweight and other body measurements in normal and growth retarded newborn infants. Lancet 1967;ii:901-5.

11 Burke G, Stuart B, Crowley P, Seanaill SD, Drumm J. Is intrauterine growth retardation with normal umbilical artery blood flow a benign condition? BMF 1990;300: $1044-5$.

12 Neligan GA, Prudham D, Steiner H. Variations in intrauterine orowth. The formative years. London: Oxford University Press, 1974

13 Marconi AM, Cetin I, Ferrazzi E, Ferrari MM, Pardi G Battaglia FC. Lactate metabolism in normal and growth Battaglia FC. Lactate metabolism in normal and

14 Nicolini U, Nicolaidis P, Fish NM, et al. Limited role of fetal blood sampling in prediction of outcome in fetal blood sampling in prediction of outcome in

15 Economidies DL, Crook D, Nicolaides KH. Hypertriglyceridemia and hypoxemia in small for gestational age fetuses. Am 7 Obstet Gymecol 1990;162:382-6.

16 Economide. Am Evans M. Pl, Nicolaides KH, Gahl WA, Bernadini I, for $\mathrm{M}$. Plasma amino acids in appropriate and smal 1989;161:1219-27.

17 Cetin I, Corbetta C, Sereni LP, et al. Umbilical amino acid concentrations in normal and growth retarded fetuses sampled in utero by cordocentesis. Am 7 Obstet Gynecol 1990;162:253-61. 